\title{
A TECHNICAL VIABILITY ANALYISIS OF USE OF THE CARAPA GUIANENSIS OIL (ANDIROBA OIL) AS CUTTING FLUID IN THE TURNING PROCESS OF THE ABNT 1020 AND 1045 STEEL GRADES
}

\author{
BOTELHO, Tamires Isabela Mesquita ${ }^{1 *}$; MONTEIRO JUNIOR, Paulo Lourenço ${ }^{1}$; DA SILVA, \\ Maria Adrina Paixão de Sousa ${ }^{1}$; DA SILVA, Rosimar Batista ${ }^{2}$; DO NASCIMENTO, Alexandre \\ Saldanha ${ }^{1}$ \\ ${ }^{1}$ Universidade Federal do Pará, Faculdade de Engenharia Mecânica, Campus Belém, , Av. Perimetral, 2651, \\ cep 66077-830, Belém - PA, Brasil \\ (fone: +559132576457 ) \\ ${ }^{2}$ Universidade Federal de Uberlândia, Faculdade de Engenharia Mecânica, Campus Santa Mônica, Av. João \\ Naves de Avila, 2121 - Bloco 1-M, cep 38400-100, Uberlândia - MG, Brasil \\ (fone: +5534 3239-4014) \\ * Autor correspondente \\ e-mail: tamiresisabela@yahoo.com.br
}

Received 22 October 2018; received in revised form 07 November 2018; accepted 10 November 2018

\section{RESUMO}

Em usinagem, o correto fluido de corte reflete em benefícios que vão desde a melhoria da qualidade da peça ao custo de fabricação. O fluido selecionado deve ainda ter características que não agrida nem o operador e nem o meio ambiente. Neste sentido, este trabalho experimental avalia os efeitos do óleo vegetal extraído da Carapa Guianensis no processo de torneamento cilíndrico externo dos aços ABNT 1020 e 1045. Foram utilizadas ferramentas de aço rápido, que foram testadas em condições de desbaste e acabamento. Além da caracterização físico-química (viscosidade dinâmica a $40^{\circ} \mathrm{C}$ e pH) do fluido, foram avaliados o comportamento do cavaco, acabamento superficial da peça e temperatura de corte das peças usinadas. Como resultado, observou-se que o fluido testado resultou em viscosidade dinâmica a $40^{\circ} \mathrm{C}$ de $0,11 \mathrm{~Pa}$.s e pH 2,3 . Os valores de rugosidade encontrados situaram-se bem próximos aqueles normalmente relatados na literatura para o torneamento de acabamento para os aços ABNT 1020 e 1045. Observou-se ainda que ao usinar o aço ABNT 1020 na condição de acabamento as temperaturas para a peça foram maiores que os demais. Dessa forma, o fluido à base da andiroba pela aplicação convencional demonstrou uma alternativa viável no processo de torneamento dos aços ABNT 1020 e 1045, pelo fato de ser biodegradável e redução dos fluidos de corte derivados do petróleo.

Palavras-chave: usinagem, fluido de corte, óleos vegetais, carapa guianensis, temperatura da peça.

\section{ABSTRACT}

In machining, the proper selection of cutting fluids result in benefits that vary from superior quality of the machined component to manufacturing cost. In addition, the must have characteristics that do not harm the user and the environment. In this sense, this work evaluates the effects of the vegetable base oil extracted from Carapa Guianensis in the external cylindrical turning process of the ABNT 1020 and 1045 steel grades. Highspeed steel tools were tested under roughing and finishing conditions. In addition to the physico-chemical characterization (dynamic viscosity at $40{ }^{\circ} \mathrm{C}$ and $\mathrm{pH}$ ) of fluid, the chip form, surface finish, tool wear and workpiece temperature were evaluated. After characterization of oil, there were recorded in the dynamic viscosity at $40^{\circ} \mathrm{C}$ of $0.11 \mathrm{~Pa}$.s and $\mathrm{pH}$ of 2.3 . The average roughness recorded were very close to those usually reported in the literature for finishing turning for ABNT 1020 and 1045 steels. It was also observed that the workpiece temperatures were higher when machining the ABNT 1020 steel grade under finishing condition than 
for other steel and conditions tested. Based on these findings, the andiroba oil delivered the conventional coolant technique demonstrated to be a technically viable alternative to conventional coolants in the turning process of ABNT 1020 and 1045 steels, because of it biodegradability characteristics and to reduce petroleumbased cutting fluids in the machining processes.

Keywords: machining, cutting fluid, vegetable oils, carapa guianensis, workpiece temperature.

\section{INTRODUÇÃO}

A usinagem dos metais ocupa uma posição de grande destaque dentro da indústria metal-mecânica e está presente em grande parte da cadeia produtiva de fabricação de componentes nas mais diversas áreas (Rios, 2005). E nos dias atuais, a abordagem do processo de usinagem leva em conta não só a seleção de parâmetros de cortes ideais, aliado ao menor custo de produção sem perda de qualidade da peça, mas também com foco na adequação e respeito às normas ambientais e saúde do operador (Encinar et. al., 2002).

Durante o processo de corte dos metais é gerado uma grande quantidade de calor, devido à energia necessária para deformação do material e à energia decorrente do atrito ferramenta- peça e cavaco-ferramenta (Diniz et al., 2010). Os fluidos de corte atuam refrigerando e lubrificando a interface peça-ferramentacavaco, como também diminuindo as altas temperaturas geradas na zona de corte. Mas o custo do fluido pode representar até $17 \%$ do custo total de fabricação. Desta forma, é preciso selecionar corretamente um fluido e sua forma de aplicação a fim de alcançar os benefícios como redução dos custos de fabricação ou aumento da taxa de produção (Machado et al., 2011), e sempre que possível observar a melhor relação benefício-custo possível.

Dentre os diversos tipos de fluidos de corte existentes, os fluidos de corte emulsionáveis, principalmente aqueles de base mineral (derivados do petróleo), ainda são os mais utilizados nas indústrias metal-mecânica devido ao custo. Esses fluidos, dependendo da sua concentração, apresentam alto poder refrigerante, fundamental para usinagem com alta velocidade de corte. Estudos realizados comparam fluidos sintéticos, semi-sintético, emulsionáveis e concluem que aqueles que utilizam emulsão são mais favoráveis que os demais para a propagação de microrganismos (Moore et al., 2000; Veillete et al., 2004). Santos e Sales (2007) relatam que os fluidos de corte possuem os nutrientes básicos (carbono, nitrogênio, enxofre e outros) para o crescimento de microrganismos. Nas operações de usinagem para a utilização de fluidos de corte emulsionáveis, faz-se a necessidade de diluir em água, para haver um balanço de refrigeração e lubrificação e normalmente a água utilizada é imprópria com a concentração de $3-15 \%$, dependendo da aplicação. Segundo Beckt et al. (2005), esse processo de diluição torna o fluido propício ao desenvolvimento de bactérias $e$ fungos, principais contaminantes da emulsão. A contaminação ocorre também através de microrganismos aderidos à poeira, partículas de aerossóis e com o próprio manuseio dos operadores por meio das bactérias presentes na pele e na mucosa, bem como a contaminação das peças e superfícies de trabalho (Passman e Rossmoore, 2010). Em grandes empresas a contaminação do fluido de corte pode levar a perda de produtividade, gastos extras com a troca do fluido de corte, problemas com a qualidade do produto acabado e reclamações dos trabalhadores por problemas de saúde, sem contar com o desgaste causando grandes problemas ambientais (Runger e Duarte, 1990).

Existe uma demanda crescente para o desenvolvimento de fluidos de corte ambientalmente amigáveis tendo como base diversos óleos vegetais, por se tratarem de matrizes obtidas de fonte renovável. A maioria dos trabalhos publicados mencionam os óleos de soja, milho e de mamona como possíveis candidatos (Grosse et al., 2016). Há um enorme potencial para a utilização de óleos vegetais como fluidos de corte no setor de fabricação (Shashidhara e Jayaram, 2010), no entanto, poucos estudos referentes aos comportamentos reológicos e tribológicos de óleos vegetais têm sido relatados na literatura específica (Gonçalves, 2013)

Os óleos vegetais são de particular interesse para o Brasil, onde existe uma grande diversidade de óleos vegetais, uma vez que o país é um dos maiores produtores mundiais desse tipo de óleo (Neves et al., 2013; Araujo et al.; 2013). Dentre os óleos vegetais, destaca-se a carapa guianensis, mais conhecida no Brasil 
como óleo da andiroba que pode ser um potencial fluido para operação de usinagem. De coloração amarelo-claro, se altera facilmente após a extração. Sua característica físicoquímica também é modificada com relação ao índice de acidez: quando exposta ao sol, o índice de acidez pode ser intensificado, podendo alcançar cerca de $90 \%$. A andiroba é uma árvore cujo óleo, extraído da semente, possui propriedades medicinais com potencial comercial e se destaca entre os óleos tradicionais no norte do país. Este óleo oferece ainda outras possibilidades de agregação de valor ao seu produto básico como sabonetes, xampus, velas e tochas repelentes (Boufleuer, 2004; Silva, 2011).

$\mathrm{O}$ estudo de materiais voltado à sua facilidade de utilização em processos de usinagem é expresso por uma grandeza chamada usinabilidade. Esta característica expressa à facilidade de usinar um material de acordo com determinado critério, mesmo não sendo uma prioridade inerente ao material. Os critérios mais usados são a vida da ferramenta, as forças de corte necessárias na usinagem e a rugosidade das peças produzidas por este processo (Ferraresi, 2011; Stemmer, 2005).

$\mathrm{Na}$ literatura Oliveira e Alves (2016) e Pusavec et al.(2010) apresentam vários estudos que descrevem os impactos negativos que os fluidos de corte contaminados podem causar ao meio ambiente e na saúde do operador. Assim, o principal objetivo desse trabalho foi analisar a viabilidade técnica do uso do óleo da carapa guianensis como fluido de corte no torneamento os aços ABNT 1020 e 1045 por meio dos parâmetros viscosidade dinâmica a $40^{\circ} \mathrm{C}, \mathrm{pH}$ diluído, forma do cavaco, acabamento superficial da peça e temperatura das peças usinadas.

\section{MATERIAL E MÉTODOS}

Foi empregada uma metodologia de ensaios utilizando um torno mecânico convencional, modelo NZ VS, do fabricante Nardini com processo de torneamento cilíndrico externo. Nos dias atuais ainda é comum realizar usinagem de peças em máquinas convencionais devido à versatilidade, baixo custo e praticidade comparadas com aquelas que são equipadas com comando numérico computadorizado (CNC). Também se empregam ferramentas de aço rápido (HSS) devido ao menor custo, inclusive faz-se necessária à etapa de reafiação. Contudo, sabe-se que estas ferramentas são menos resistentes ao calor durante a usinagem e apresentam desgaste acelerado quando comparadas as ferramentas de metal duro, o que limitam o seu emprego em situações com elevadas velocidades de corte. As ferramentas utilizadas no experimento foram de aço rápido (HSS) com $12 \%$ Co.

Os materiais das peças testadas no trabalho foram no total 4 (quatro) barras cilíndricas de aço ABNT 1020 e 1045, 02 (duas) para cada aço com dimensões iniciais iguais a: $23 \mathrm{~mm}$ de diâmetros por $38 \mathrm{~mm}$ comprimento, todos com um furo no centro nas extremidades, representados na figura 1.

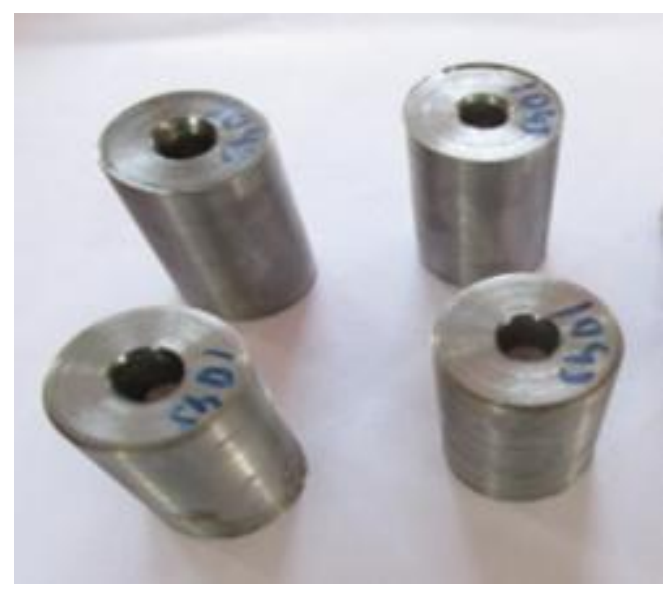

Figura 1. Corpo-de-prova dos aços ABNT 1020 e 1045

A composição química de cada material e dureza são apresentadas nas tabelas 1 e 2, respectivamente.

Tabela 1. Valores da composição química dos aços

\begin{tabular}{c|c|c|c|c|c}
\hline $\begin{array}{c}\text { Aço } \\
\text { ABNT }\end{array}$ & $\mathbf{C} \%$ & Mn\% & Si\% & $\begin{array}{c}\text { P\% } \\
\text { (max) }\end{array}$ & $\begin{array}{c}\text { S\% } \\
\text { (max) }\end{array}$ \\
\hline 1020 & 0,22 & 0,44 & 0,21 & 0,04 & 0,05 \\
1045 & 0,44 & 0,71 & 0,20 & 0,04 & 0,05 \\
\hline
\end{tabular}

Tabela 2. Dureza dos aços

\begin{tabular}{c|c}
\hline Aço ABNT & HB \\
\hline 1020 & $127 \pm 0,5$ \\
1045 & $174 \pm 0,5$ \\
\hline
\end{tabular}

Os parâmetros de entrada utilizados neste trabalho foram: velocidade de corte (Vc), avanço (f) e profundidade (ap) e os valores de cada um são apresentados na Tabela 3. O fluido de corte, óleo da carapa guianensis, foi coletado de forma artesanal no município de Igarapé- 
Mirim (PA), sendo este material rejeito do processo de fabricação artesanal da região. O fluido foi aplicado na zona de corte via a técnica convencional por inundação (vazão de 630,93 $\mathrm{ml} / \mathrm{s}$ ) e na direção (sobrecabeça, como ilustrado na Figura 2).

A viscosidade dinâmica da carapa guianensis foi medida usando um viscosímetro do fabricante Hake à temperatura de $40^{\circ} \mathrm{C}$, que foi mantida constante durante o experimento utilizando um cilindro coaxial modelo VT-550, segundo a norma ASTM D 2770-4. Já a análise do $\mathrm{pH}$ foi realizada com pHmetro portátil digital da marca HI221.

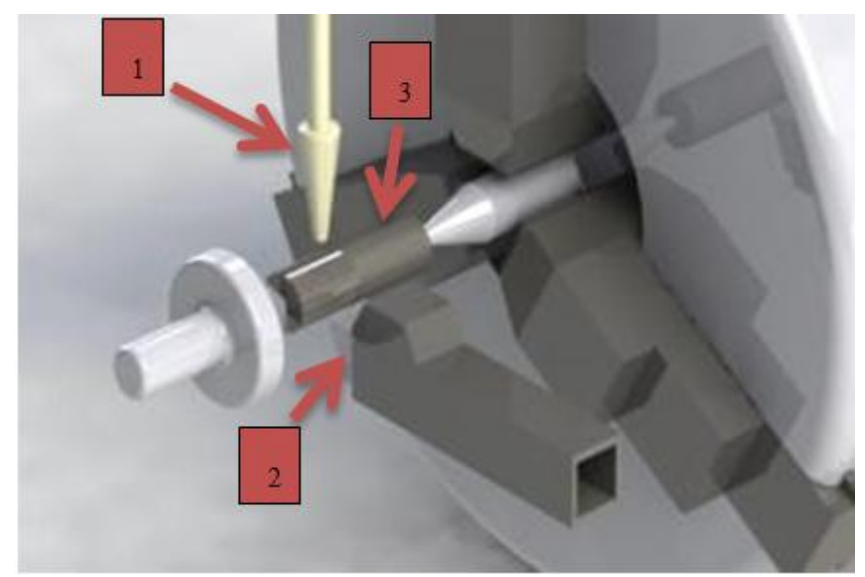

Figura 2. Detalhe da montagem do sistema ferramenta-peça, com a seta 1 indicando o bocal, seta 2 a ferramenta de corte e a seta 3 a disposição da peça entre pontas

O processo de torneamento se deu do inicio ao fim do percurso do corpo de prova, ou seja, um único passe. Sendo após a finalização de cada ensaio trocada a peça, ferramenta de corte e os parâmetros definidos na tabela 3. As variáveis de saída analisadas foram:

- Formas de Cavaco: Nos ensaios de usinagem, após o primeiro e o último passe da ferramenta da usinagem de cada corpo-de-prova foram coletados os cavacos resultantes, os quais foram analisados e classificados de acordo com a norma ISO 3685.

- Rugosidade: a obtenção da rugosidade média aritmética $(\mathrm{Ra})$ foi realizada através de um rugosímetro portátil, com resolução $0,01 \mathrm{~mm}$, modelo TR200 da marca Time. Foram adotados um cut-off de $0,8 \mathrm{~mm}$ e comprimento de amostragem $4 \mathrm{~mm}$, de acordo com a norma NBR ISO 4287. Para todas as medições foram feitas quatro ao longo da seção longitudinal e em extremidades opostas e retirada uma média dos valores obtidos.

- Temperatura da peça: A técnica de medição empregada neste trabalho foi aquela proposta por Silva, et.al. na qual se utiliza uma termocâmera da marca FLIR que é, posicionada de maneira que os pontos de corte permanecessem direcionados na zona do corte, conforme mostra a Figura 3, a uma distância de $800 \mathrm{~mm}$ desta zona, menor que a distância de resolução máxima desta que é de $1000 \mathrm{~mm}$. As aquisições dos dados foram feitas por meio da interface do computador com o software FLIR Tool® dos dados térmicos da termocâmera.

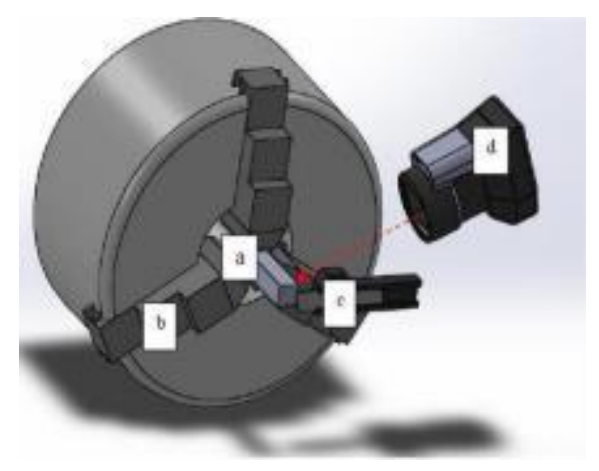

Figura 3. Amostra (a) afixada na castanha do torno (b), a ferramenta de aço rápido (c), na posição de corte e o termômetro (d) posicionado para aquisição de dados térmicos

\section{RESULTADOS E DISCUSSÃO}

\subsection{Viscosidade Dinâmica a $40^{\circ} \mathrm{C} \mathrm{e} \mathrm{pH}$}

Geralmente, as propriedades físicas e químicas dos fluidos de corte são modificadas com o tempo, devido ao longo período de uso, a forma inadequada de utilização e manutenção ou ainda pelas ações de microrganismos, podendo causar riscos nos ambientes de trabalho e naturais (quando são descartados). Sendo assim, é de grande importância o estudo da dinâmica dos fluidos nestes ambientes (Bhattacharya et al., 2004; Helena et al., 2001). Muniz (2008) relata que a estabilidade das emulsões de um fluido de corte pode ser afetada pela qualidade da água. Esta, portanto, devem estar isenta de impurezas, microrganismos e excesso de cloro.

A viscosidade deve ser suficientemente baixa, de modo a permitir uma fácil circulação do fluido e alta o suficiente para que se tenha uma boa aderência do fluido às superfícies da ferramenta (Souza, 2009), sendo que esta última favorece a função lubrificação e atua redu-zindo 
o atrito na interface peça-ferramenta, o que implica em redução das forças de corte e manutenção da rugosidade em níveis mais baixos. Quanto maior a necessidade de lubrificação, maior a vantagem de utilização de fluidos de corte de base vegetal em relação às demais bases, independente da condição de corte empregada (Gonçalves, 2013). Contudo, segundo Silva (2000), quanto menos viscoso for o fluido de corte melhor será a operação de usinagem.

O valor de $\mathrm{pH}$ dos fluidos é um forte indicativo do nível de ataque das suas propriedades anticorrosívas, pois além do consumo de emulgadores e agentes anticorrosivos, as bactérias geram subprodutos ácidos, resultantes de seu metabolismo, reduzindo o $\mathrm{pH}$ da emulsão e a proteção anticorrosiva (Boufleuer, 2004). No presente estudo a análise de $\mathrm{pH}$ resultou uma média de 2,3. $\mathrm{O}$ pH é o principal indicador para avaliação do fluido (Marcelino, 2013). Para Runge e Duarte (1990) o valor satisfatório do pH de uma emulsão para o uso é acima de 8,7. Os autores Santos e Sales (2007), Lima (2012) e Takahashi (2012) citam que o valor 7,8 e 8,7, exige a adição de biocidas para controlar o grau de contaminação e correção de $\mathrm{pH}$ com agentes alcalinizantes. O valor do óleo da andiroba está abaixo do valor ideal de uma emulsão, pois é um óleo graxo de origem vegetal puro sem aditivos e encontra-se próximo dos valores encontrado nas literaturas de Melo da Silva et al.(2014) e Vasconcelos et al. (2008).

\subsection{Formas do Cavaco}

Na Figura 3 (a) a (d) são apresentadas as imagens obtidas dos cavacos gerados para os diferentes aços testados nas diferentes condições de corte investigadas. Quanto aos cavacos gerados e apresentados, observa-se que eles assumiram diferentes formas e tipos: forma tubular emaranhada Fig. 3(a) e longo Fig. 3(b). Na Fig3(c) em tubular curta e na Fig3(d) helicoidal longa.

Por se tratar de dois metais com diferentes teor de carbono e dureza, houve uma diferença de comportamento do cavaco, com uma mudança discreta de tipo tubular no aço ABNT 1020 para tipo helicoidal do aço ABNT 1045. Segundo Ferraresi (2011), a forma mais conveniente é a helicoidal, sendo o cavaco em lascas preferido em casos onde o cavaco deve ser removido pelo fluido de corte ou quando há pouco espaço disponível para o cavaco. Um ca- vaco longo, em forma de fita, além de se enrolar sobre a peça sendo usinada, danificando-a, pode atingir o operador, machucando-o seriamente, além de o cavaco longo ser muito mais difícil de manipular e requerer um volume muito maior para armazenamento que um cavaco curto com o mesmo peso (Diniz et a.l, 2010).

Amâncio et al. (2013) avaliaram as variáveis que interferem no processo de usinagem em um torno CNC utilizando o método de planejamento e experimento Taguchi onde

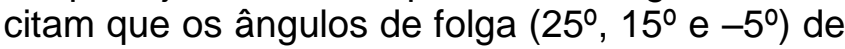
ferramentas de aço rápido HSS são fatores que influenciam na forma dos cavacos, no caso do ângulo de posição ou folga, quanto maior este ângulo em relação ao cabo da ferramenta, maior será a largura do cavaco, provocando uma superfície de contato maior, isto solicita um esforço maior da ferramenta para retirada de cavaco. E o ângulo de inclinação quando for negativo, pode gerar um péssimo acabamento superficial da peça.

\subsection{Rugosidade}

Na Figura 4 são mostrados os valores parâmetro $\mathrm{Ra}(\mu \mathrm{m})$, obtidos dos corpos de prova para as condições de acabamento e desbaste dos aços ABNT 1020 e 1045.

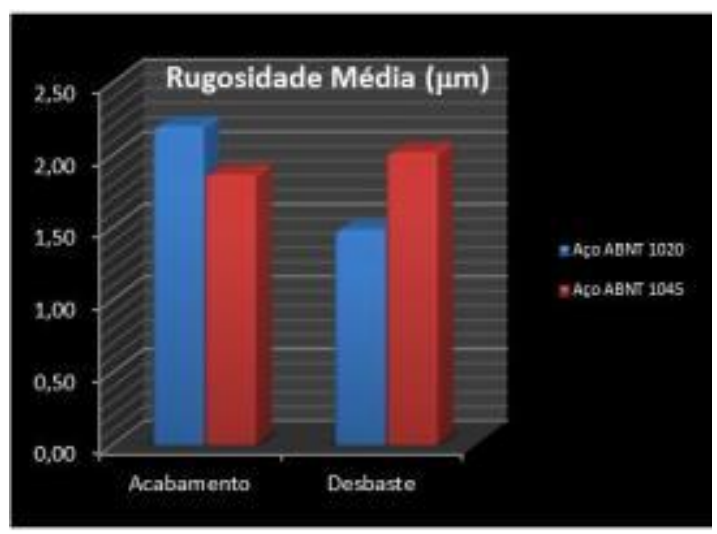

Figura 4. Rugosidade média das peças em aços ABNT 1020 e 1045 condições de acabamento e desbaste

Quanto aos valores de rugosidade, da Figura 4 observa-se que praticamente não há diferença nos valores ao usinar na condição de acabamento comparando os dois aços. Observase que para o aço ABNT 1045 obteve-se uma menor rugosidade, entretanto, para a condição de desbaste, o aço ABNT 1020 apresentou um decréscimo mais significativo, ou seja, um melhor acabamento superficial. Diversos estudos foram 
feitos visando à otimização do torneamento em vários materiais e a sua influência sobre o acabamento superficial e 0 desgaste da ferramenta.

Um estudo da minimização do custo de usinagem por torneamento de aço ABNT 52100, utilizando-se a metodologia de superfície de resposta foi realizado por Mendes et al. (2007), considerando a vida da ferramenta e o acabamento superficial da peça. Seus resultados indicam que a minimização do custo é obtida com as maiores velocidades de corte utilizadas no seu experimento, com maiores taxas de remoção de material. Araújo et al. (2013) avaliaram 0 desempenho do óleo refinado vegetal de coco babaçu (Orbignya Oleifera) como fluido de corte, aplicado via técnica da mínima quantidade de fluido - MQF, com diferentes vazões, considerando os esforços de usinagem e 0 acabamento superficial, durante 0 processo de fresamento frontal do aço ABNT 1045 , e relataram que a técnica MQF reduziu as forças de usinagem pela sua função lubrificante e o atrito na interface cavaco-ferramenta. Com isso, eles demonstraram que o óleo de coco babaçú resultou menores quando comparado ao fluido LB 2000 (fluido comercial) e menores quando comparado com a condição a seco (o mesmo não ocorreu com relação à rugosidade média).

\subsection{Temperatura da Peça}

Nas Figuras 5 e 6 são mostrados os valores de temperatura da peça, aço ABNT 1020 e aço ABNT 1045, respectivamente, nas diferentes condições de corte testadas.

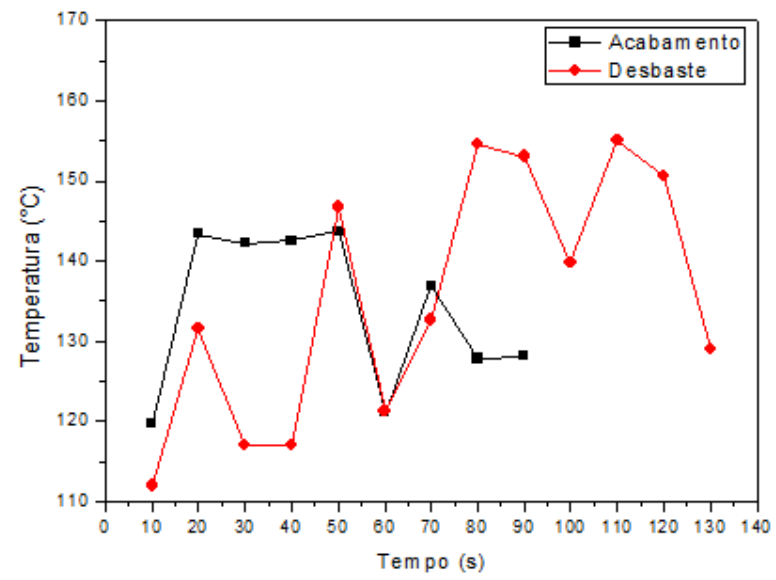

Figura 5. Temperatura da peça com progressão da usinagem do aço ABNT 1020 em condições de acabamento e desbaste

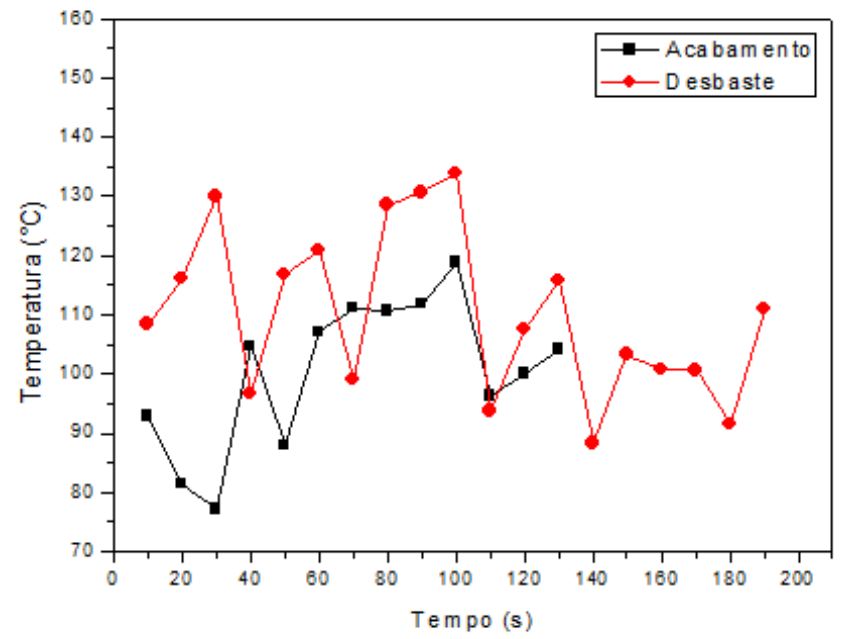

Figura 6. Temperatura pelo tempo do aço ABNT 1045 em condições de acabamento e desbaste

Em relação a temperatura da peça após a usinagem dos aços ABNT 1020 e 1045, observase que a usinar o aço ABNT 1020 os valores de temperatura foram maiores na condição de desbaste, comportamento este que se assemelhou para o aço ABNT 1045. Nas condições de desbaste, em ambos os aços, a aplicação com o óleo da andiroba proporcionou temperaturas relativamente altas dadas as dimensões dos corpos-de-prova usinados e os parâmetros adotados. Machado et al. (2011) relata que, o calor no plano de cisalhamento pode até ser benéfico para a usinagem, pois aumenta a temperatura nas zonas primárias e secundárias e diminui a resistência mecânica do material da peça para dar continuidade à deformação plástica (efeito de amolecimento). O calor gerado na zona secundária de cisalhamento tem maior influência sobre o desempenho da ferramenta e é praticamente fator limitante para a taxa de remoção de material. Em especial, por meio do aumento da velocidade de corte, o contato entre a superfície de folga da ferramenta e a peça (zona terciária) pode ocorrer no mesmo caso de ferramentas sem desgaste de flanco e, além disso, também pode contribuir para o aumento da temperatura da peça e da ferramenta. O óleo da andiroba atuou como lubrificante não sendo eficaz na redução da temperatura, ou seja, não atuando de forma a reduzir a área de contato na interface cavaco-ferramenta e fazendo com que o cavaco escoe com mais facilidade (Souza et al., 2010).

Suarez et al. (2009) informam que a temperatura de usinagem também influencia no 
controle dimensional e no acabamento superficial da peça. O aumento da temperatura contribui ainda para reduzir as forças e a potência de usinagem. Sabe-se que a interface de contato entre a ferramenta e a peça trabalho no processo de usinagem na superfície de flanco está sujeita a pressões elevadas e consequentemente a altas temperaturas (Tönshoff, 2004). Durante o processo de torneamento, observou-se que houve várias oscilações de temperatura para todas as condições. Tais resultados já são conhecidos da literatura (Guimarães, 2014; Haddag e Nouari, 2013) e prováveis justificativas são que próximo ao final da área de corte uma menor quantidade de calor é dissipada e por conta do aumento progressivo do desgaste da ferramenta ao longo de cada ensaio (Silva e Wallbank, 1999).

\section{CONCLUSÕES}

Com base nos ensaios experimentais realizados pôde-se observar o comportamento do óleo da carapa guianensis como fluido de corte no processo de torneamento dos aços ABNT 1020 e 1045, nas condições de acabamento e desbaste concluindo que:

- O óleo da carapa guianensis apresentou valores para a análise da viscosidade dinâmica a $40^{\circ} \mathrm{C}$ de 0,11 Pa.s. Para a análise de $\mathrm{pH}$ o resultado adquirido foi de 2,3;

- Os valores de rugosidade média, Ra, foram inferiores a 2,2 $\mu \mathrm{m}$, valores bem próximos daqueles relatados na literatura para aços comum ao carbono usinados com bits de HSS em condições semelhantes as investigadas nesta pesquisa;

- Na condição de acabamento para o aço ABNT 1020, a aplicação da andiroba proporcionou maiores temperaturas de peça.

Considerando que as características avaliadas se encontram de acordo com a literatura para fluidos de corte, a carapa guianensis se mostrou viável para utilização com essa finalidade.

\section{AGRADECIMENTOS}

Agradecemos a Base Naval de Val-deCans (BNVC), pelo espaço experimental, ao Instituto Federal Pará (IFPA), pelas análises e ao Conselho Nacional de Desenvolvimento Científico e Tecnológico (CNPq).

\section{REFERÊNCIAS}

1. ASTM D (2004), 2770-04: Standard practice for calculating viscosity index from kinematic Viscosity at 40 and 100. Annual Book of ASTM Standards, ASTM International, West Con-shohocken.

2. Amâncio, D.A., Toledo, J.V., Ferreira, J.R.; Melo, M.L., Silva, G., Figueira, D.S.; Estudo e aprimoramento dos ângulos da geometria da ferramenta de aço rápido para o torneamento do nióbio em torno cnc utilizando o método de Taguchi. $7^{0}$ Congresso Brasileiro de Engenharia de Fabricação, Penedo, Itatiaia, RJ, Brasil, 15-19 de abril de 2013.

3. Araujo, A.S., Machado, A.R., Neves, T.E.S., Paiva., A.E., Rodrigues, J.R.P.; Esforços de usinagem e acabamento superficial no fresamento frontal do aço abnt 1045 com aplicação mqf do óleo refinado vegetal de coco babaçu. $7^{\circ}$ Congresso brasileiro de engenharia de fabricação, Penedo, Rio de Janeiro, Brasil, 20-24 de maio de 2013.

4. Becket, W.,Kallay, M., Sood, A., Zuo, Z., Milton, D. Hypersensitivity pneumonit-is associated with environmental mycobacteria. Environ Health Perspect. USA, v. 113, n 6, p. 767-770, Fev. 2005.

5. Bhattacharya, M., John, J., Raynor, P. C. Emulsions containing vegetable oils for cutting fluid application. Colloids and Surfaces A: Physicochem. Eng. Aspects, v. 237, p. 141-150. 2004.

6. Boufleuer, N.T. Aspecto ecológico de andiroba (carapa guianensis aublet., meliaceae), como subsidio ao manejo e conservação. Dissertação (mestrado) Universidade Federal do Acre, Rio Branco, AC, Brasil, 2004.

7. Diniz, A.E., Marcondes, F.C., Coppini N.L. Tecnologia da usinagem dos materiais 7.ed. São Paulo: Artliber, 2010. 262p.

8. ENCINAR, J. M. et al. Biodiesel Fuels from Vegetable Oils: Trans esterification of Cynara cardunculus L. Oils with Ethanol. Energy \& Fuels, v. 16, n. 2, p. 443-450. 2002.

9. Ferraresi, D. Fundamentos da Usinagem dos Metais. 15 ed. São Paulo: Blucher, 
Janeiro, 2011. p 102.

10. Gonçalves, J.F.S. Proposição de método de desenvolvimento de fluido de corte de base vegetal. Tese de Doutorado, Instituto Tecnológico da Aeronáutica, São José dos Campos, São Paulo, Brasil, 2013.

11. Grosse, T., Winter, M., Baron, S., Hoffmeister, H., Herrmann, C., Droder, K. Honing with polymer based cutting fluids. CIRP Journal of Manufacturing Science and Technology. p. 89-98, 2015.

12. Guimarães, C. B. Análise da usinabilidade da liga Al-7\%Si solidificada em um sistema unidirecional horizontal. Dissertação (mestrado), Universidade Federal do Pará, Pará, Brasil, 2014.

13. Haddag, B., Nouari, M. Tool wear and heat transfer analyses in dry machining based on multi-steps numerical modelling and experimental validation. Wear. 302 , 1158-1170, 2013.

14. SILVA, M. B., WALLBANK, J., Cutting temperature: prediction and measurement methods-a review. Journal of Materials Processing Technology. 88(1-3): 195-202, 1999

15. Helena W., Rolf. L., Theo, M. Lubricant base fluids based on renewable raw materials their catalytic manufacture and modification. Appl Catal A: Gen, v. 221, p. 429-42. 2001.

16. ISO (1993) 3685: Tool Life Testing with Single-Point Turning Tools. ISO International Organization for Standardization.

17. Lima, E.A. A vida pode aumentar, Dependendo da Formulação. Revista Máquinas e Metais. Editora aranda, Setembro. 2012.

18. Machado, Á.R., Abrão, A.M., Coelho, R.T.; Da Silva, M.B. Teoria da Usinagem dos Materiais. 2.ed. São Paulo - SP: Editora Edgard Blucher, 2011. 397p.

19. Marcelino, L.I. O. Fluido de corte mineral emulsionável: monitoramento, análise da degradabilidade, reformulação e estratégias de aumento de tempo de vida no processo de retificação. Dissertação (mestrado) - Centro Federal de Educação Tecnológica de Minas Gerais, Belo
Horizonte, MG, Brasil, 2013.

20. Melo da Silva, P.M, Da Silva, D.P, De Souza, E.C, Dos Silva, A.S, Muller, R.C.S Caracterização físico-químico de óleo de andiroba comercializado em Belém-Pa e em Bragança. $54^{\circ}$ Congresso Brasileiro de Quimica. Natal, Rio Grande o Norte, Brasil, 3 - 7 de outubro de 2014.

21. Mendes, R.R.A, Paiva, A.P, Ferreira, J.R., Estudo da minimização do custo de usinagem por torneamento do aço ABNT 52100 utilizando-se a metodologia de superfície de resposta. 4ํㅡㄹ Congresso Brasileiro de Engenharia de Fabricação. Águas de São Pedro, SP, Brasil, 15-18 de abril de 2007.

22. Moore, J.S., Christensen, M., Wilson, R.W., Wallace Jr.,R.J., Zhang, Y., Nash, D.R., Shelton, B. Mycobacterium Contamination of Metalworking Fluids: Involvement of a Possible New Taxon of Rapidly Growing Mycobacteria. AlHAJ. USA, v. $61, n^{\circ} 2$, p. 205-13, abr. 2000

23. Muniz, C.A.S. Novas formulações de fluido de corte: otimização, propriedades e recuperação de óleo usado. Tese de Doutorado. Universidade federal do Rio Grande do Norte, RN, Natal, Brasil, 2008.

24. NBR ISO (2002) 4287: Especificações geométricas do produto (GPS) Rugosidade: Metódo do perfil - Termos, definições e parâmetros da rugosidade. NBR - Norma Brasileira. Interna-tional. ISO - Organization for Standardization

25. Neves, T.E.S., Bacci, M., Araujo, A.S., Rodrigues, J.R.P., Paiva, A.E.; Efeito do óleo de canola refinado e das condições de corte na força de avanço na furação do aço abnt 1045 . $7^{\circ}$ Congresso brasileiro de engenharia de fabricação, Penedo, Rio de Janeiro, Brasil, 20-24 de maio de 2013.

26. Oliveira, J.F.G.,ALVES, S.M. Adequações ambiental dos processos usinagem utilizando produção mais limpa como estratégia de gestão ambiental. $<$ http://en.scienctificcommons.org/salete martins alves.> Acessado em 02 fevereiro 2016.

27. Passman, F.J., Rossmoore, H.W. Reassensing the health risks associated with em-ployee exposure to MWF 
microbes. Lubricat Engineering. USA, v. 58, n 7, p. 30-38, mai. 2002.

28. Pusavec, F., Krajnik, P., Kopac, J. Transitioning to sustainable production part l: application on machining technologies. Journal of Cleaner Production, v. 18, p.174-184. 2010.

29. Rios, M. R. S., Desempenho de emulsão leitosa e fluido sintético na furação de aço inoxi-dável. Revista Máquinas e Metais, São Paulo, Jul, 2005.

30. Runge, P. R. F., Duarte, G. N. Lubrificantes nas Indústrias. Fluidos de Corte. Cotia: Triboncept, cap.4, p.71-172. 1990.

31. Santos, S.C., Sales, W.F. Aspectos tribológicos da usinagem dos materiais. 1.ed.São Paulo: Artliber Editora, 2007. $246 p$.

32. Shashidhara, Y.M., Jayaram, S.R. Vegetable oils as a potential cutting fluid an evolution. Tribology International, v. 43 p. 1073-1081. 2010.

33. Silva, J. V. M., et al.,; Necking test methodology for solidific alloys in directional solidifi-cation devices. XIV Congresso do SBPMAT, 1286-32, Rio de Janeiro, RJ, Brasil, 27/09-01/10/2015.

34. Silva, D.A.L., Soares, N.L., Fernandes, G.K.O., Santana M. F. S., Correa, A. P. P. Caracterização Fisico-Quimica de Óleos de Andiroba Extraidos nos Municipios do Acre e Amazonas. $51^{\circ}$ Congresso Brasileira de Quimica. São Luiz, Maranhão, Brasil, 09-13 de outubro de 2011.

35. Silva, E. J. Análise da influência dos tipos de fluido de corte e rebolo na retificação do aço SAE HVN-3. Dissertação (mestrado) - Universidade Estadual de São Paulo, Bauru, SP, Brasil, 2000.

36. Sousa, J.A., Aguiar, F.M., Sousa, M.N., Mundim, P.O., Cardoso, R.N., Machado, A.R. Investigação Experimental da Temperatura de Usinagem no Corte Ortogonal de Alumínio. VI Congresso Nacional de Engenharia Mecânica, Campo Grande, Pernambuco, Brasil, 1821 de Agosto de 2010.

37. Souza, J. Avaliação de fluidos de corte de base vegetal na furação de ferro fundido vermicular. Dissertação (mestrado) Instituto Tecnológico da Aeronáutica, São Paulo, SP, Brasil, 2009.

38. Stemmer, C.E. Ferramentas de Corte I. Editora da UFSC, 6ª Edição, Florianópolis, 2005.

39. Suarez, M. P., Costa, E.S., Machado, A.R., Abrão, A.M., Precisão Dimensional em Canais Usinados na Liga de Alumínio 7075 - T7. V Congresso Brasileiro de Engenharia de Fabricação, Belo Horizonte, MG, Anais em CD Rom, Artigo: 011072262, 14-17 de Abril de 2009.

40. Takahashi, D.F. Nova metodologia para otimizar biocidas em fluido de corte. Revista Máquinas e Metais, junho. 2012.

41. Tönshoff, T., Ben Amor, R., Pereira, A. A., Boehs L. Análise da integridade da superfície de uma ferramenta de metal duro. III Congresso Nacional de Engenharia Mecânica, Belém, PA, Brasil, 10-13 de agosto de 2004.

42. Vasconcelos, M.A.M., Mattietto, R.A., Gongalvez, A.C.S., Oliveira, P.S., Moreira, P.I.O., Alves, S.M., Moreira, D.K.T., Figueiredo, J.G., Dantas, H.A. Avaliação do processo de extração e caracterização do óleo e sementes de andiro-ba (Carapa guianensis Aublet). In: Conferência do subprograma de Ciência e Tecnologia, PPG7, Belém, 2008.

43. Veillete, M., Thorne, P.S., Gordon, T.;Duchaine, C. Six Month Tracking of Microbial Ghowth in a Metalworking Fluid After Sytem Cleaning and Recharging. Ann Occup Hyg. Canadá, v. 48, n 6, p. 541-546, Agosto. 2004. 
Tabela 3. Resumo dos parâmetros operacionais utilizados no ensaio de torneamento nas condições de desbaste e acabamento dos aços ABNT 1020 e 1045

\begin{tabular}{|c|c|c|c|c|c|}
\hline \multirow{3}{*}{ Aço ABNT } & \multicolumn{5}{|c|}{ Condição } \\
\hline & \multicolumn{5}{|c|}{ Desbaste } \\
\hline & $\begin{array}{l}\text { Ângulo } \\
\text { de folga } \\
(\alpha 0)\end{array}$ & $\begin{array}{l}\text { Ângulo de } \\
\text { saída }(\gamma 0)\end{array}$ & $\begin{array}{l}\text { Profundidade } \\
\text { de corte } \\
(\mathrm{mm})\end{array}$ & $\begin{array}{c}V_{c} \\
(\mathrm{~m} / \mathrm{min})\end{array}$ & $\begin{array}{c}\mathrm{f} \\
(\mathrm{mm} / \mathrm{ver})\end{array}$ \\
\hline 1020 & $20^{\circ}$ & $44^{\circ}$ & 0,5 & 345 & 0,053 \\
\hline \multirow[t]{2}{*}{1045} & $20^{\circ}$ & $44^{\circ}$ & 0,5 & 206 & 0,053 \\
\hline & \multicolumn{5}{|c|}{ Acabamento } \\
\hline 1020 & $2^{\circ}$ & $46^{\circ}$ & 0,5 & 409 & 0,053 \\
\hline 1045 & $2^{\circ}$ & $46^{\circ}$ & 0,5 & 298 & 0,053 \\
\hline
\end{tabular}

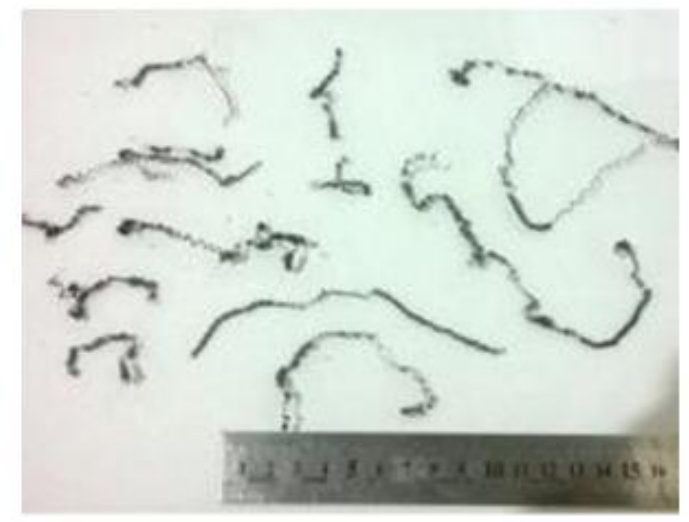

(a)

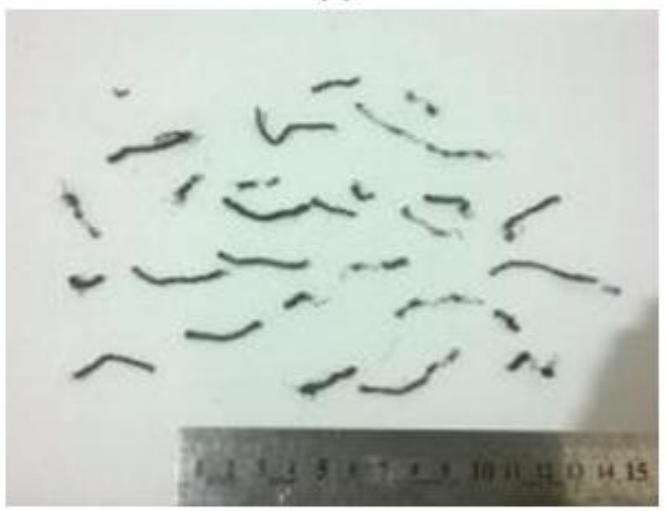

(c)

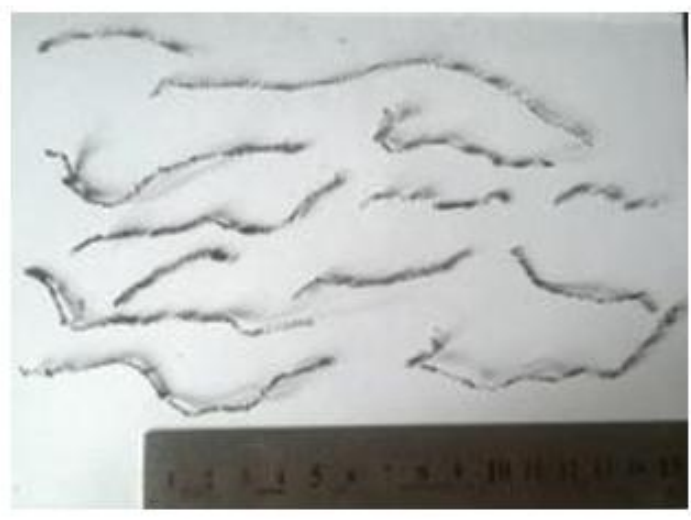

(b)

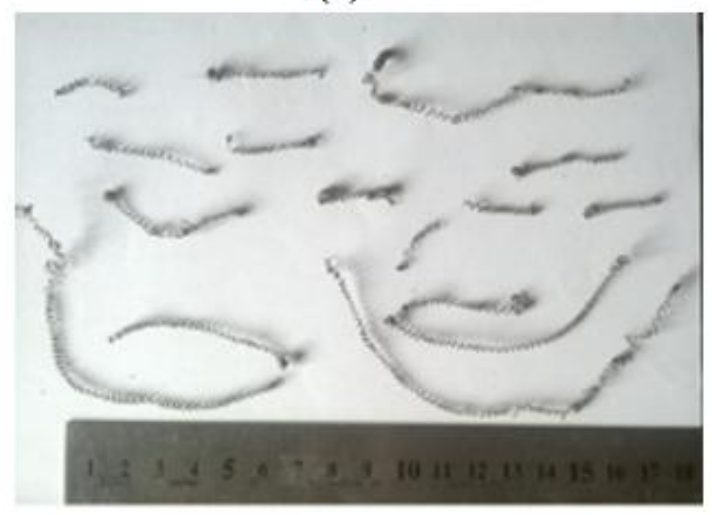

(d)

Figura 3. Cavaco gerado no torneamento, (a) aço ABNT 1020 - acabamento, (b) aço ABNT 1045 acabamento, (c) aço ABNT 1020 - desbaste e (d) aço ABNT 1045-desbaste 